\title{
Efficacy and safety of auricular acupressure for chemotherapy- induced peripheral neuropathy among patients with breast cancer: a study protocol for a randomized controlled trial
}

\author{
Mi Sook Jung ${ }^{1}$, Jin Sun $\mathrm{Lee}^{2}$, Eun Hee Sohn ${ }^{3}$, Kyeongin Cha ${ }^{1}$, Nondumiso Satiso Dlamini ${ }^{1}$, Mijung Kim ${ }^{1}$ \\ ${ }^{1}$ College of Nursing, Chungnam National University, Daejeon, Korea; ${ }^{2}$ Department of Surgery and Research Institute for Medicinal Sciences, \\ College of Medicine, Chungnam National University, Daejeon, Korea; ${ }^{3}$ Department of Neurology, Chungnam National University Hospital, \\ Daejeon, Korea \\ Correspondence to: Mijung Kim. College of Nursing, Chungnam National University, 266 Munhwa-ro, Jung-gu, Daejeon 35015, Korea. \\ Email: cipnmj@gmail.com.
}

Background: Chemotherapy-induced peripheral neuropathy (CIPN) is a frequently-reported distress symptom in breast carcinoma patients under chemotherapy. Although previous studies emphasized lack of ideal neuroprotective or therapeutic agents for CIPN, there are no strongly recommended treatments. Nevertheless, auricular acupressure (AA) is a novel remedy for controlling symptoms in many healthcare settings. However, therapeutic effects of AA among patients with CIPN have not yet been elucidated fully. Therefore, we designed a trial to examine the effectiveness and safety of AA in breast cancer patients.

Methods: This randomized, double-blind, sham-controlled trial will assess 120 breast cancer survivors. After enrollment, the participants will be stratified depending on administration of medications prescribed for CIPN treatment, and then assigned randomly to the experimental or control groups in an allocation ratio of 1:1. For experimental groups, AA will be applied on four points, namely, shemen, liver, spleen, and finger/ toe, while for the control groups, sham AA will be exerted on other four points that are remote from the treatment points and unrelated to neuropathic symptoms. All participants will undergo the same acupressure procedure, for two minutes each time, thrice daily for 3 weeks, and CIPN symptoms and health-related quality of life will be assessed by a blinded research assistant and a physician before, during, immediately after, and 4 weeks after AA. Occurrence, type, and severity of safety issues will be routinely monitored to confirm the non-toxic nature of AA. Repeated measures analysis of variance will be used to examine the changing pattern of CIPN symptoms and based on sensitivity analysis.

Discussion: It is expected that the rationale and design of this protocol will offer knowledge regarding a standardized process to guide current and future studies and accumulates clinical experiences in applying non-pharmacological intervention. The present trial is the first to examine the therapeutic effects of AA in breast cancer patients with CIPN. The findings of the study may provide convincing evidence regarding the effectiveness of CIPN symptoms.

Trial Registration: Clinical Research Information Service, Republic of Korea, ID: KCT0004930. Registered retrospectively on April 14, 2020.

Keywords: Peripheral neuropathy; ear; acupressure; clinical trial protocol; randomized controlled trial (RCT)

Submitted Jul 23, 2020. Accepted for publication Nov 17, 2020.

doi: 10.21037/apm-20-1469

View this article at: http://dx.doi.org/10.21037/apm-20-1469 


\section{Introduction}

Chemotherapy-induced peripheral neuropathy (CIPN) is generally defined as damage to the peripheral nerves caused by toxicity of chemotherapeutic agents such as platinumbased antineoplastic agents, vinca alkaloids, epothilones, taxanes, proteasome inhibitors and immunomodulatory drugs $(1,2)$. CIPN is characterized mainly by changes in sensory function that may be accompanied by deficits in motor and autonomic function such as numbness, tingling sensation, altered sensation, allodynia, weakness, and dysesthesias (3). These neuropathic symptoms can affect functional abilities in daily living and lead to difficulties in continuing chemotherapy which may be associated with a negative effect on overall survival $(4,5)$. According to the National Comprehensive Cancer Network Task Force Report, taxanes as part of first-line therapy for early-stage and metastatic breast cancer were associated with a high incidence of CIPN in $57-83 \%$ of patients treated with paclitaxel and $11-64 \%$ of individuals receiving docetaxel (6). Although its prevalence has decreased over time, at least $28 \%$ of patients continue to experience neuropathic symptoms at 6 months or more after completing chemotherapy $(3,7,8)$.

The lack of ideal neuroprotective or therapeutic agents for CIPN is emphasized as a key unmet need among cancer patients and healthcare professionals (9). For example, duloxetine is the only symptomatic treatment for CIPN recommended by the American Society of Clinical Oncology based on sufficient evidence; however, its therapeutic effect is modest (10). Various non-pharmacological management modalities have been developed as better alternatives in complementary and integrative medicine (11). A recently published systematic review and meta-analysis showed that acupuncture, therapeutic massage, foot bath, and exercise have been used most frequently to reduce neuropathic symptoms and improve the health-related quality of life (12). Among these interventions, acupuncture therapy is a wellaccepted therapeutic modality used to relieve cancer- and treatment-related symptoms as well as other peripheral neuropathic symptoms, possibly due to the lack of known serious side effects, effective combinations with other treatments, and alterative way to help patients who cannot receive pharmacological treatments $(13,14)$. However, the needle insertion method of acupuncture is also known to be a barrier that cannot completely be free from safetyrelated issues such as infectious adverse events following acupuncture (15).
Auricular acupressure (AA), which was derived from auricular acupuncture used in traditional Chinese medicine, is a noninvasive method of applying physical pressure to acupuncture points. It has been used as a low-risk complementary treatment to relieve symptoms for which needle insertion acupuncture has already proven to yield clinical benefits (16). Previous studies support the clinical applicability of AA in the effective management of medical conditions such as menstrual migraines (17), chronic low back pain (18), acute postoperative pain (19), insomnia and daytime dysfunction (20-23), and overweight (24). A recently published interventional study that assessed changes in CIPN symptoms among 13 cancer patients showed that AA yielded beneficial effects in CIPN management (with a $38-48 \%$ reduction in symptom intensity compared to pretreatment) and maintained a similar degree of long-term effects until after 1 month (25).

The potential mechanism of AA is based on Paul Nogier's findings of an inverted fetus on the auricle representing a somatotopic map of the human body onto the ear, reported in 1956. According to his concept, each specific point of the entire auricular surface corresponds to specific organs and regions of the body (26). Although two studies have examined changes in brain activity corresponding to auricular acupuncture points by using neuroimaging techniques such as functional magnetic resonance imaging, there is scientific evidence to supports a somatotopic connection between auricular points and body structures $(27,28)$. A recently published functional magnetic resonance imaging study also revealed that auricular point pressure can directly stimulate auricular areas innervated by both the trigeminal and vagus nerves and, consequently, could lead to alterations in brain connectivity and functional modification of the central pain processing pathway of the brain (29). From this point of view, AA can have beneficial effects on CIPN symptom management through stimulating both the auriculo-vagal afferent pathway, hence reducing pain perception in the brain, and the efferent vagus nerve by stimulating the cholinergic anti-inflammatory pathway, thereby controlling the extent of the inflammatory response (1,30-32). Taken together, it has been suggested that AA may be a useful, non-pharmacological, and noninvasive approach that yields beneficial effects regarding symptom management in individuals with CIPN.

To the best of our knowledge, only three consecutive studies, conducted by the same research group, have evaluated the effects of AA on CIPN management using 
a one-group pretest-posttest design $(25,29,33)$. More evidence obtained from randomized controlled trials (RCTs) is needed to translate these findings into clinical practice. The present study aims to assess the feasibility of AA in the management of CIPN and to evaluate changes in CIPN symptoms and patients' livelihoods while undergoing taxanes-based treatment for non-metastatic cancer of the breast. We present the protocol in accordance with the SPIRIT reporting checklist (available at http://dx.doi. org/10.21037/apm-20-1469).

\section{Methods}

\section{Study design and setting}

The present study is a randomized, double-blind, shamcontrolled trial. The experiment will be conducted at a single large-volume territory hospital, the largest hospital in the central region of South Korea. Trained researchers will screen female patients who are newly diagnosed with taxane-induced peripheral neuropathy after receiving chemotherapy for breast cancer. Eligible participants will be stratified by the current status of administration of pharmacological agents (gabapentinoids and duloxetine) in the treatment of CIPN and randomly allocated in a 1:1 ratio to the experimental $\mathrm{AA}$ group or the sham AA group. All participants will undergo AA for three weeks and follow-up at 4 weeks (Figure 1). The protocol of the present study has been reviewed and approved by the Institutional Review Board of Human Research of the Chungnam National University (approval No. 201906SB-088-01). Informed consent developed in accordance with the Declaration of Helsinki will be obtained from all individual participants before starting any data collection in this study. The protocol fulfills the populated Standard Protocol Items: Recommendations for Interventional Trials (SPIRIT) checklist (Figure 2) and the checklist for items in Standards for Reporting Interventions in Clinical Trials of Acupuncture (STRICTA) (additional Table S1). This study has been registered at the Clinical Research Information Service (cris.nih.go.kr; KCT0004930).

\section{Study participants}

A total of 120 patients will be recruited at the Cancer Center of the Chungnam National University Hospital. All patients who underwent chemotherapy within a year for breast cancer will be selected as potentially eligible subjects through screening by the researcher. After screening, we will invite patients for enrollment according to the inclusion and exclusion criteria listed in Table 1.

\section{Sample size estimation}

The sample size was calculated based on the primary outcome. According to a single study that evaluated AA as intervention for CIPN, an overall change of 3.0 points in mean scores between pre- and post-intervention assessment was observed, with a significant decrease in the worst pain score, which was referred to in the study (25). However, the aforementioned study was conducted by using a one-group pretest-posttest design and no other studies could yield data to compute standardized variations connecting two means obtained from two groups. Accordingly, the sample size was calculated considering the highest number of participants required in pilot studies and the general formulas for superiority design in RCTs (34). To identify changes in scores between pre- and post-intervention periods in the present study, the null and the alternative hypotheses are H0: $d_{P}-d_{B}=0$ and $H a: d_{P}-d_{B}>0$, respectively $\left(d_{B}\right.$ : baselinescore, $\mathrm{d}_{\mathrm{p}}$ : post-score, and $\delta$ : clinical admissible margin of superiority). Based on the study hypotheses, 24 patients per group would be needed to attain a power of $80 \%$ for detecting a variation between groups with a two-sided level of significance less than 0.05 (see below). Considering a possible dropout rate of 0.2 , a total of 120 patients (30 patients in each group) are needed.

$$
\begin{aligned}
& n=2 \times\left[\frac{Z_{1} \frac{\alpha}{2}+Z_{1}-\beta}{\delta}\right]^{2} \times s^{2} \\
& n=2 \times\left[\frac{1.96+0.845}{3}\right]^{2} \times 3.64^{2} \\
& \mathrm{n}=23.17
\end{aligned}
$$

\section{Randomization and blinding}

A randomization code list was generated by Sealed Envelope Ltd. and assessed on June 7, 2019, by a person who is not involved in the present study. Participants will be stratified based on the current status of administration of pharmacological agents to prevent confounding effects which can influence the outcome variables. They will then 


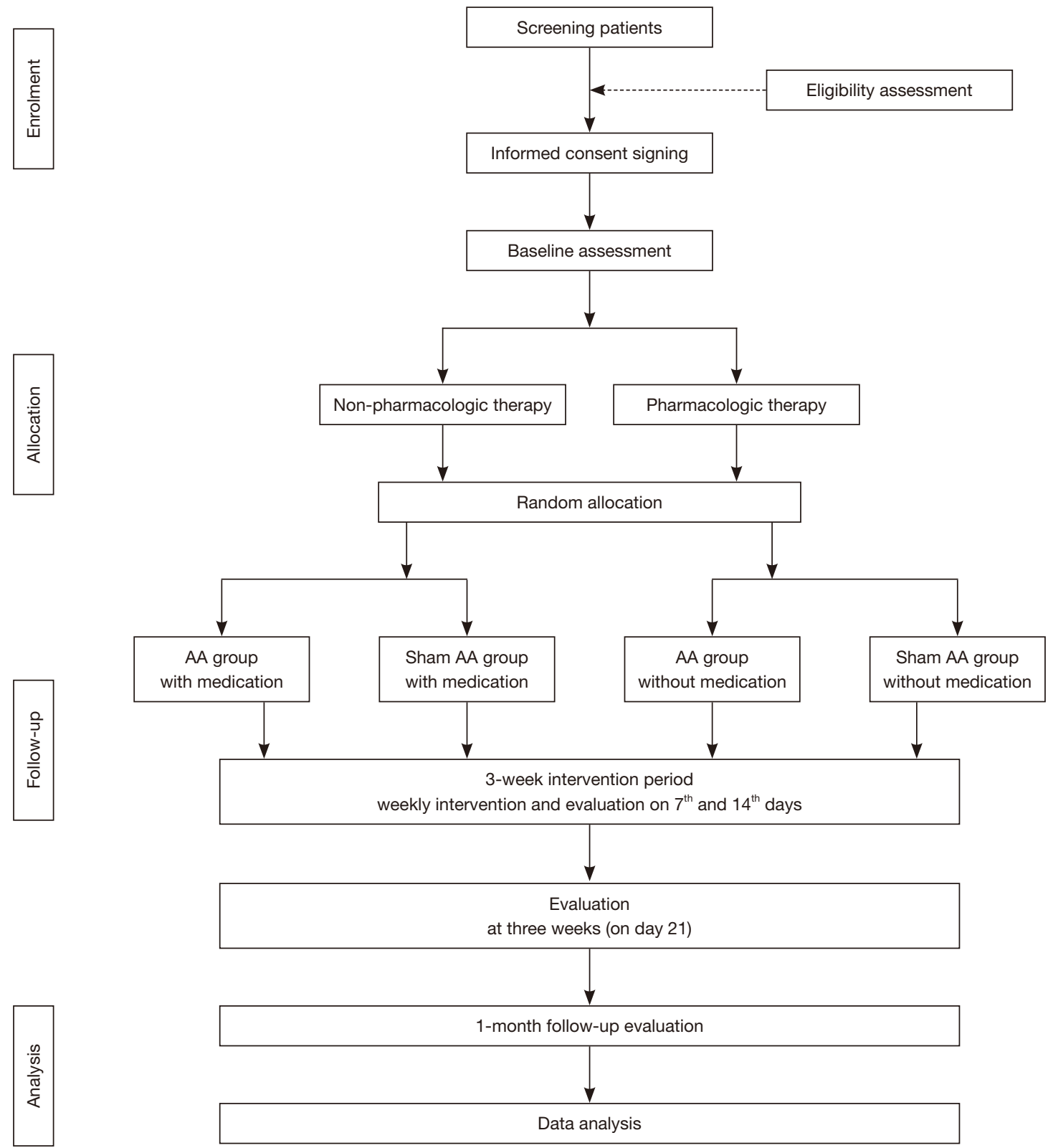

Figure 1 Flowchart of the patient selection process.

be assigned randomly to two arms in allocation sequence in a 1:1 ratio: medication AA group (experimental group 1), non-medication AA group (experimental group 2), medication-sham AA group (control group 1), and nonmedication-sham AA group (control group 2), with 4 groups in all. Each group will contain an equal number of participants. To prevent researcher bias from confounding the results, each member of the study team will be blinded to the randomization code, allocation, and equivalent treatment except for one who will perform the intervention.

\section{Intervention}

All participants will undergo AA on the unilateral ear for three weeks. At each visit, the following procedures will be performed: (I) check the ear to identify the target points based on the protocol; (II) sterilize the entire external ear with 75\% isopropyl alcohol; and (III) attach Vaccaria seeds 


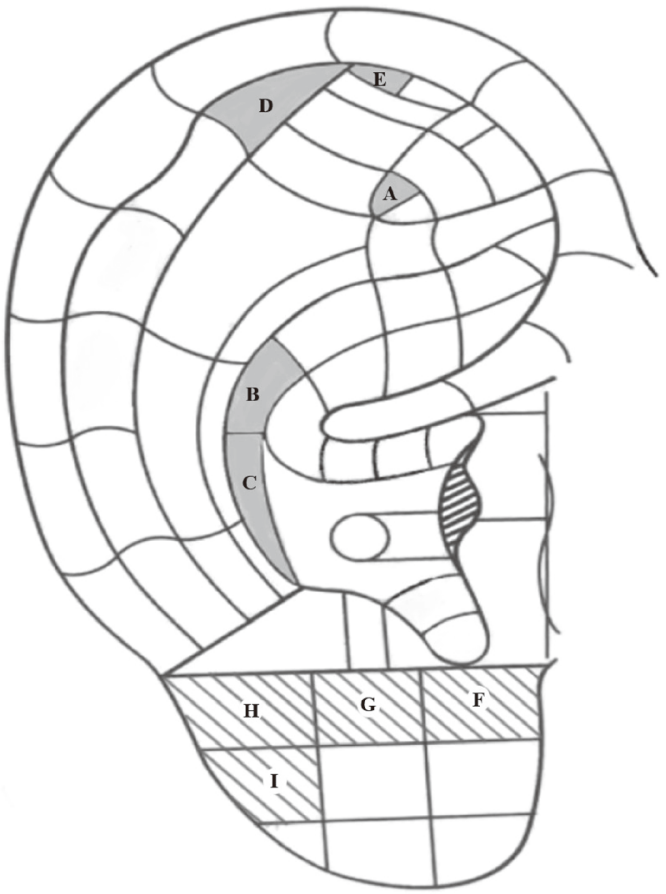

Figure 2 Locations of specific acupressure points. A, shenmen; B, liver; C, spleen; D, finger; E, toe; F, tooth; G, tongue; H, jaw; I, internal ear.

(with diameters of approximately $0.2 \mathrm{~cm}$ ) covered using waterproof adhesive tape (with dimensions of approximately $0.7 \mathrm{~cm} \times 0.7 \mathrm{~cm})$ in the target auricular points. The Vaccaria seed is a natural botanical seed that does not have any medical benefits or harmful effects on health. The person who will perform these procedures will instruct the participants to apply steady pressure on the taped seed attached to the ear points until maximal tolerable discomfort is sensed. Each procedure will take about 2 minutes with pressing on each target auricular point for at least one second in each trial and a two-minute pause between two auricular pressings. All participants will be instructed to repeat the pressings themselves thrice daily for five consecutive days and then to remove the taped seeds from the ear on the morning of the sixth day. Two consecutive days will be excluded to prevent the well-known side effect of treatment (contact dermatitis) that can be induced by the long-term use of adhesive tapes. New seeds will be replaced according to a weekly schedule including the day of visit, five days of application, and two days off. The participants will be advised to call the researcher immediately when the seeds or tapes are detached from the ear. Regardless of the groups, each trial participant will record each performance and event during the intervention period in a diary.

\section{Experimental groups}

Participants in two AA groups (experimental groups 1 and 2) will undergo AA treatment at every visit once a week for three weeks. The auricular points selected for this study include those of the shenmen, liver, spleen, toe, and finger. The shenmen, liver, and spleen points will be used in common for all experimental groups and one of either the toe or finger points will be used depending on the region at which pain is felt by the participant. The rationales for selecting these points are based on literature reviews and the clinical recommendations of three of the Korean AA educators. Specifically, the shenmen has explicitly been described as a master point and has been used to manage symptoms such as sleep disturbance and pain $(25,35)$. The liver point stores and promotes "Qi" circulation and the spleen point regulates blood supply and flow. Thus, both the liver and spleen points contribute to the improvement metabolism which reduces the neurotoxic effects of chemotherapeutic agents. The toe and finger points are the direct reflex points of symptomatic body parts according to the physical reflex theory and will be used to relieve physical symptoms after chemotherapy (Figure 2).

\section{Control groups}

Participants in two sham AA groups (control groups 1 and 2) will undergo AA treatment on sham points of the ear. Four sham points will be used, including those of the tooth, tongue, jaw, and internal ear. These auricular points are clearly remote from the treatment points and are unrelated to neuropathic symptoms (36) (Figure 2).

\section{Recruitment and data collection}

Potential participants will be invited by a skilled researcher to ensure that standard procedures and guidelines for data collection are observed. Simultaneously, advertisements will be also posted in the research site to improve the rate of recruitment. Details of the study will be provided to those who are interested in this clinical trial. Individuals who meet the inclusion and are willing to participate will be finally enrolled after providing written informed consent. As shown in Table 2, participants will enter the intervention within a week after completing the baseline assessment. Additional assessment will be performed once a week during 
Table 1 Patients' eligibility criteria

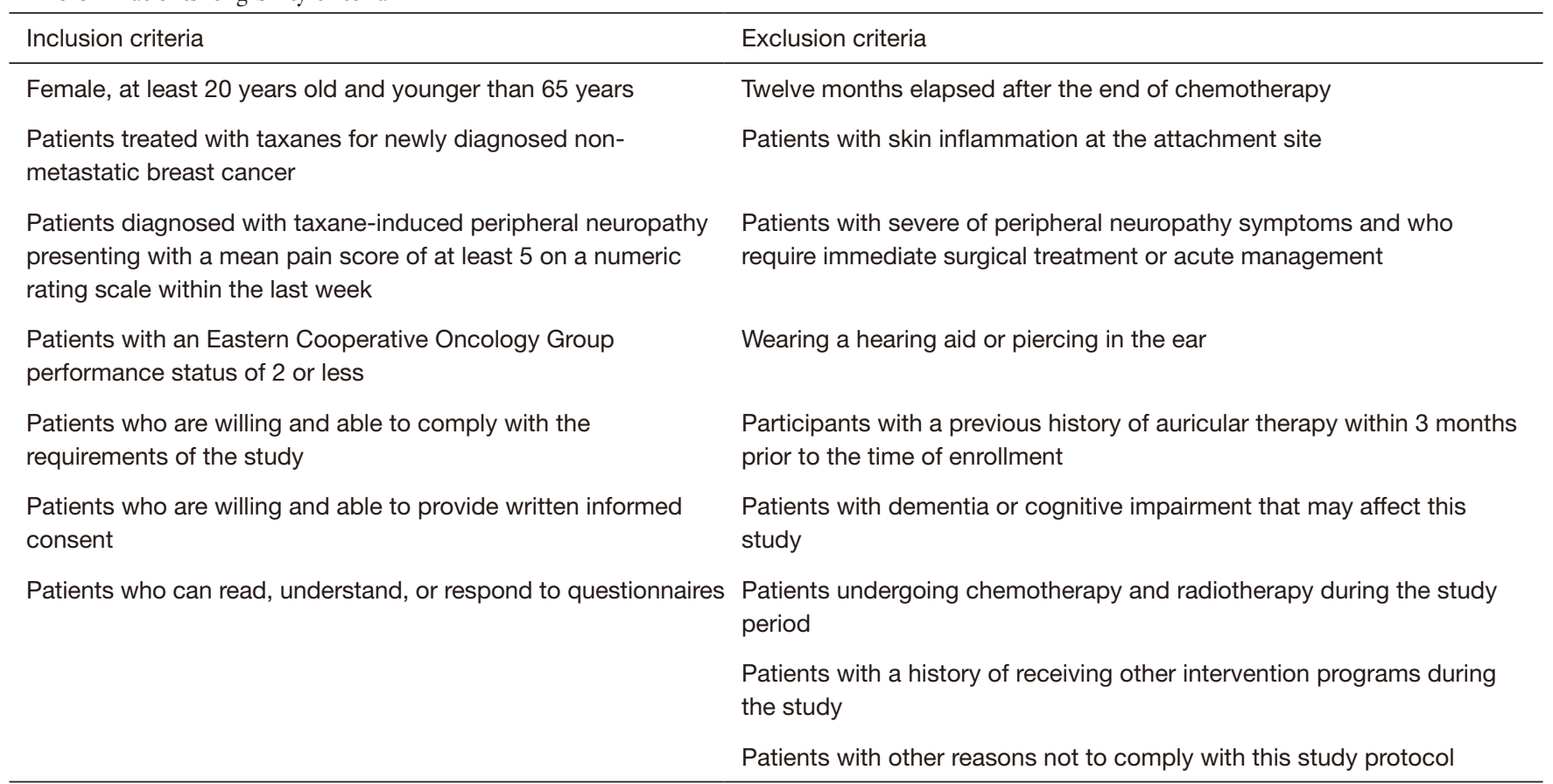

Table 2 Study timeline according to the SPIRIT checklist

\begin{tabular}{|c|c|c|c|c|c|c|c|c|}
\hline & \multicolumn{8}{|c|}{ Study period } \\
\hline Timepoint & $-t_{1}$ & 0 & 0 & Day $1 \pm 3$ & 1 week \pm 3 & 1 week \pm 3 & 1 week \pm 3 & 1 week \pm 3 \\
\hline \multicolumn{9}{|l|}{ Enrolment } \\
\hline Eligibility screen & $x$ & & & & & & & \\
\hline Demographics & $x$ & & & & & & & \\
\hline Medical history & $x$ & & & & & & & \\
\hline Allocation & & $x$ & & & & & & \\
\hline \multicolumn{9}{|l|}{ Interventions } \\
\hline \multicolumn{9}{|l|}{ Assessments } \\
\hline NRS & $x$ & & $\mathrm{x}$ & $X^{*}$ & $x$ & $x$ & $x$ & $x$ \\
\hline TNS & & & $x$ & $X^{*}$ & $x$ & $x$ & $x$ & $x$ \\
\hline EORTC QLQ-CIPN 20 & & & $x$ & $X^{*}$ & $x$ & $x$ & $x$ & $x$ \\
\hline FACT-G & & & $x$ & & & & $x$ & \\
\hline
\end{tabular}

*, assessments on day 1 can be omitted when collecting data on the same day as the baseline. NRS, Numeric Rating Scale; TNS, Total Neuropathy Score; EORTC QLQ-CIPN 20, European Organization for Research and Treatment of Cancer Quality of Life Chemotherapy Induced Peripheral Neuropathy 20; FACT-G, Functional Assessment of Cancer Therapy-General. 
the intervention period. A follow-up assessment will be performed on the day of completion of the AA intervention and 4 weeks later.

\section{Outcome measures}

The outcome measures are presented in Table 2.

\section{Numeric rating scale (NRS)}

The NRS will be used to estimate the overall intensity of CIPN symptoms. This measure is one of the most widely used clinical tools and known for its easy applicability. Participants will respond by rating the intensity of symptoms on an 11-point scale ranging from 0 (no symptoms) to 10 (worst possible symptoms). The validity of the NRS has been confirmed in studies of patients with cancer $(37,38)$.

\section{Total neuropathy score (TNS)}

The TNS is a physician-based evaluation method used to assess CIPN symptoms. The TNS will be scored on a range of 0-40 (each on a scale of 0-4) by a physician through interview and several tests including sensory, motor, autonomic symptoms, pin sensibility, vibration sensibility, strength, tendon reflexes, vibration threshold, thermal threshold, sural amplitude, and peroneal amplitude (39). We will use 9 items (except for sural amplitude) because there is no electrodiagnostic machine at the center of the hospital. The psychometric properties of the scale were determined in previous studies of patients with neuropathy caused by chemotherapy $(40,41)$.

European Organization for Research and Treatment of Cancer Quality of Life (EORTC-QLQ) ChemotherapyInduced Peripheral Neuropathy 20 (CIPN 20)

The EORTC-QLQ-CIPN20 will be used to assess the symptoms of CIPN and functional limitations related to these symptoms. This measure comprises sensory, motor, and autonomic domains. Participants will be asked to assess the severity of symptoms and interference with functioning by using a 4-point Likert scale ranging from 1 (not at all) to 4 (very much). Increasing scores indicate worse CIPN states (42). The original measure includes 20 items, 19 of which will be used in the present study because one item applies to male respondents. This measure is a valid and reliable tool for evaluating CIPN symptoms and functional limitations $(43,44)$.

\section{Functional Assessment of Cancer Therapy-General (FACT-G)}

The FACT-G will be used to assess health-related quality of life. This measure includes four subscales on wellbeing: the physical (7 items), social/family (7 items), emotional (6 items), and functional (7 items) domains. Each item is rated on a 5 -point Likert scale ranging from 0 (not at all) to 4 (very much) (45). The FACT-G has been used worldwide and is confirmed to be a reliable and valid instrument $(46,47)$.

\section{Data management and monitoring}

Data obtained from participants will be encoded using a unique identifier and recorded. The data will then be stored in both an electronic database and a locked cabinet. Only permitted researchers can access and manage the data. Checks for logic will be performed to identify missing data and inconsistencies. The whole process of this trial will be conducted under the supervision of monitors that include researchers, ethics committee, and data supervisors.

\section{Safety and withdrawals}

Patients will be interviewed for intervention and assessment at each meeting for intervention and assessment, as well as over telephone calls and regular text messaging between visits. We will enquire about adverse events, including their occurrence and severity related to this trial. If any adverse event (skin damage, infection, and allergic reaction in the ear with AA treatment) occurs, the research team of this trial will provide the corresponding treatment to the participant. The principal investigator will immediately report any adverse events to the ethics committee in the authors' institution. A safety analysis will be performed to assess the incidence of adverse events and the number of patients who experience adverse events in each group. Participants will be free to withdraw from the trial at any time and for any reason. Withdrawals will be documented, and an active follow-up will be conducted to monitor the occurrence of any adverse events throughout the study period.

\section{Data analysis}

Statistical analyses will be carried out by a statistician who is blinded to the random allocation using SPSS version 24 (IBM Corporation, Armonk, NY, USA). To improve 
sensitivity of outcomes, all data will be analyzed through two approaches using a 5\% significance level. An intentionto-treat analysis that includes all participants who receive the intervention at least once will be performed and missing data will be handled using the last-observation-carriedforward method. Since the trial was designed to confirm the effectiveness of AA, an additional per-protocol analysis was performed, which includes only participants who complete the entire trial, and excludes participants who violate the protocol. Baseline outcomes in the experimental and control groups will be compared using an independent $t$-test or Wilcoxon rank sum test depending on the distribution of the data. Analysis of covariance will be used to assess the mean differences while controlling for a current status of administration of pharmacological agents. Changes in mean scores from the baseline to follow-up assessment in each group will be computed to assess the effect of the intervention. To examine the interaction between groups and time, repeated-measures analysis of variance will be performed. Clinically relevant covariates will be included in these analyses.

\section{Discussion}

Chemotherapy-induced neuropathic symptoms are commonly reported by patients who have received chemotherapeutic regimens, including taxanes such as paclitaxel (Taxol) and docetaxel (Taxotere) (48). Since taxane was introduced in the 1990s, it has been used as a representative drug in combination with anthracyclines and alkylating agents to treat breast cancer, and has led to an improvement in the survival rate (49). To date, the mechanism underlying CIPN has not yet been elucidated fully. Possible mechanisms underlying the function of taxane include blocking of axonal transport by the microtubule, which is important for neuronal survival and function $(50,51)$. A previously published review identified taxane as a risk factor for CIP and reported differences in incidence rates and severity depending on whether taxane was used or not (52). We also verified whether the cumulative dose of taxane, a physiologic factor, directly affected the occurrence of CIPN symptoms among Korean breast cancer survivors using a structural equation model (53). Based on these findings, the present study will focus on a group of patients treated with taxane-based chemotherapy that should be investigated for the risk of developing CIPN.

In the present study, we will schedule treatment sessions on a weekly basis, including 2 days off between weeks for
3 weeks. There are two reasons for this treatment schedule. First, the safety is an important issue in an interventional study. We found that the probability of adverse events may increase if the AA is performed for longer than 1 week. Hence, the 2-day pause between weekly treatments will enable recovery from auricular stimulations caused by the pressure and prevent the risk of skin damage, infection, and any allergic reactions caused using attached tapes and materials (36). This treatment schedule will be an effective way of improving safety. Second, an appropriate intervention period is considered regarding feasibility. In previous studies, acupuncture interventions were performed over various periods (ranging from 4 to 12 weeks) in managing CIPN symptoms $(25,54-57)$. To the best of our knowledge, only one study applied a 4-week AA intervention to cancer patients with CIPN symptoms (25). Yeh $e$ al. suggested that a 3-week plan for AA therapy may be more feasible due to the schedule of chemotherapy (58). Thus, the inclusion criteria of the present study do not identify the end of chemotherapy but only consider whether chemotherapy is performed during the intervention period. Although the total number of treatment cycles varies depending on the regimen prescribed, chemotherapy with docetaxel is generally administered once every 3 weeks $(59,60)$. Considering of the treatment schedule applied in clinical settings, a 3-week AA intervention was determined. A recently published study of the trend in auriculotherapy application in the last 10 years in Korea also supports the fact that our intervention has no considerable barriers in applicability regarding the entire period and weekly schedule of AA intervention (61).

In the present study, we will invite patients who receive pharmacological agents to reduce CIPN symptoms, as well as their counterparts who do not take any medications. Controlling for the current administration of pharmacological agents may contribute to the clear elucidation of the effect of intervention on CIPN; however, ethical concerns may exist. Our approach can help researchers improve the rate of participation and adherence and conduct the natural setting trial in clinical practices (62).

Various studies have confirmed the role of AA in managing symptoms related to cancer and treatment, as well as CIPN $(25,58,63,64)$. However, there are research gaps regarding study design, sample size, presence of control groups, and controlling confounding variables (65). To develop a well-designed trial, we considered the following aspects: (I) applying a random allocation method to prevent selection of bias, the Hawthorne effect, and 
the placebo effect, (II) including a sham control group to minimize the placebo effect, and (III) assessing the study outcomes by using both clinician-administered and selfreported measures. Our study would likely add knowledge required to fill the gaps in research. This protocol offers a standardized process to guide current and future studies and accumulates pieces of evidence regarding clinical experiences in applying non-pharmacological interventions. Since the present study seeks to investigate the effectiveness and safety of AA, the findings are expected to provide convincing evidence in these regards for breast cancer patients with CIPN. Moreover, the results will provide healthcare professionals with lessons for managing CIPN symptoms.

\section{Acknowledgments}

Funding: MK was supported by the National Research Foundation of Korea grant funded by the Korea government (grant number: 2017RA2B4004328) and the Brain Korea 21 Plus project of the National Research Foundation of Korea Grant. This funding source will not have any role including design, execution analyses, interpretation of the data, and decision of this study.

\section{Footnote}

Reporting Checklist: The authors have completed the SPIRIT reporting checklist. Available at http://dx.doi.org/10.21037/ apm-20-1469

Conflicts of Interest: All authors have completed the ICMJE uniform disclosure form (available at http://dx.doi. org/10.21037/apm-20-1469). The authors have no conflicts of interest to declare.

Ethical Statement: The authors are accountable for all aspects of the work in ensuring that questions related to the accuracy or integrity of any part of the work are appropriately investigated and resolved. The present study was reviewed and approved by the ethics committee of the Chungnam National University (approval No. 201906SB-088-01). Informed consent developed in accordance with the Declaration of Helsinki will be obtained from all individual participants before starting any data collection in this study.

Open Access Statement: This is an Open Access article distributed in accordance with the Creative Commons Attribution-NonCommercial-NoDerivs 4.0 International License (CC BY-NC-ND 4.0), which permits the noncommercial replication and distribution of the article with the strict proviso that no changes or edits are made and the original work is properly cited (including links to both the formal publication through the relevant DOI and the license). See: https://creativecommons.org/licenses/by-nc-nd/4.0/.

\section{References}

1. Zajączkowska R, Kocot-Kępska M, Leppert W, et al. Mechanisms of chemotherapy-induced peripheral neuropathy. Int J Mol Sci 2019;20:1451.

2. Staff NP, Grisold A, Grisold W, et al. Chemotherapyinduced peripheral neuropathy: A current review. Ann Neurol 2017;81:772-81.

3. Seretny M, Currie GL, Sena ES, et al. Incidence, prevalence, and predictors of chemotherapy-induced peripheral neuropathy: A systematic review and metaanalysis. Pain 2014;155:2461-70.

4. Bhatnagar B, Gilmore S, Goloubeva O, et al. Chemotherapy dose reduction due to chemotherapy induced peripheral neuropathy in breast cancer patients receiving chemotherapy in the neoadjuvant or adjuvant settings: a single-center experience. SpringerPlus 2014;3:366.

5. Zanville NR, Nudelman KN, Smith DJ, et al. Evaluating the impact of chemotherapy-induced peripheral neuropathy symptoms (CIPN-sx) on perceived ability to work in breast cancer survivors during the first year posttreatment. Support Care Cancer 2016;24:4779-89.

6. Stubblefield MD, Burstein HJ, Burton AW, et al. NCCN task force report: management of neuropathy in cancer. J Natl Compr Canc Netw 2009;7 Suppl 5:S1-S26; quiz S27-8.

7. Eckhoff L, Knoop A, Jensen MB, et al. Persistence of docetaxel-induced neuropathy and impact on quality of life among breast cancer survivors. Eur J Cancer 2015;51:292-300.

8. Bao T, Basal C, Seluzicki C, et al. Long-term chemotherapy-induced peripheral neuropathy among breast cancer survivors: prevalence, risk factors, and fall risk. Breast Cancer Res Treat 2016;159:327-33.

9. Fradkin M, Batash R, Elmaleh S, et al. Management of peripheral neuropathy induced by chemotherapy. Curr Med Chem 2019;26:4698-708.

10. Colvin LA. Chemotherapy-induced peripheral neuropathy: 
where are we now? Pain 2019;160 Suppl 1:S1-10.

11. Samuels N, Ben-Arye E. Integrative approaches to chemotherapy-induced peripheral neuropathy. Curr Oncol Rep 2020;22:23.

12. Oh PJ, Kim YL. Effectiveness of non-pharmacologic interventions in chemotherapy induced peripheral neuropathy: A systematic review and meta-analysis. J Korean Acad Nurs 2018;48:123-42.

13. Li K, Giustini D, Seely D. A systematic review of acupuncture for chemotherapy-induced peripheral neuropathy. Curr Oncol 2019;26:e147-54.

14. Lu W, Rosenthal DS. Acupuncture for cancer pain and related symptoms. Curr Pain Headache Rep 2013;17:321.

15. Xu S, Wang L, Cooper E, et al. Adverse events of acupuncture: a systematic review of case reports. Evid Based Complement Alternat Med 2013;2013:581203.

16. Weaver MT. Acupressure: an overview of theory and application. Nurse Pract 1985;10:38-9, 42.

17. Sun LH, Li XH, Li WL, et al. Body acupuncture combined with auricular acupressure for menstrual headache: a randomized controlled clinical trial. Zhen Ci Yan Jiu 2015;40:70-4.

18. Yang LH, Duan PB, Hou QM, et al. Efficacy of auricular acupressure for chronic low back pain: A systematic review and meta-analysis of randomized controlled trials. Evid Based Complement Alternat Med 2017;2017:6383649.

19. Zhong Q, Wang D, Bai YM, et al. Effectiveness of auricular acupressure for acute postoperative pain after surgery: A systematic review and meta-analysis. Chin J Integr Med 2019;25:225-32.

20. King HC, Spence DL, Hickey AH, et al. Auricular acupuncture for sleep disturbance in veterans with posttraumatic stress disorder: a feasibility study. Mil Med 2015;180:582-90.

21. Lan $Y, W u X$, Tan HJ, et al. Auricular acupuncture with seed or pellet attachments for primary insomnia: a systematic review and meta-analysis. BMC Complement Altern Med 2015;15:103.

22. Yang JL, Zhang R, Du L, et al. Clinical observation on the neurotransmitters regulation in patients of insomnia differentiated as yang deficiency pattern treated with warm acupuncture and auricular point sticking therapy. Zhongguo Zhen Jiu 2014;34:1165-8.

23. Kober A, Scheck T, Schubert B, et al. Auricular acupressure as a treatment for anxiety in prehospital transport settings. Anesthesiology 2003;98:1328-32.

24. Huang CF, Guo SE, Chou FH. Auricular acupressure for overweight and obese individuals: A systematic review and meta-analysis. Medicine (Baltimore) 2019;98:e16144.

25. Yeh CH, Lukkahatai N, Campbell C, et al. Preliminary effectiveness of auricular point Acupressure on chemotherapy-induced neuropathy: Part 1 self-reported outcomes. Pain Manag Nurs 2019;20:614-22.

26. Nogier R. How did Paul Nogier establish the map of the ear? Med Acupunct 2014;26:76-83.

27. Romoli M, Allais G, Airola G, et al. Ear acupuncture and fMRI: a pilot study for assessing the specificity of auricular points. Neurol Sci 2014;35 Suppl 1:189-93.

28. Alimi D, Geissmann A, Gardeur D. Auricular acupuncture stimulation measured on functional magnetic resonance imaging. Med Acupunct 2002;13:18-21.

29. Yeh CH, Caswell K, Pandiri S, et al. Dynamic brain activity following auricular point acupressure in chemotherapy-induced neuropathy: A pilot longitudinal functional magnetic resonance imaging study. Glob Adv Health Med 2020;9:2164956120906092.

30. Hou PW, Hsu HC, Lin YW, et al. The history, mechanism, and clinical application of auricular therapy in traditional Chinese medicine. Evid Based Complement Alternat Med 2015;2015:495684.

31. He W, Wang X, Shi H, et al. Auricular acupuncture and vagal regulation. Evid Based Complement Alternat Med 2012;2012:786839.

32. Kavoussi B, Ross BE. The neuroimmune basis of anti-inflammatory acupuncture. Integr Cancer Ther 2007;6:251-7.

33. Yeh CH, Lukkahatai N, Campbell C, et al. Preliminary effectiveness of auricular point acupressure on chemotherapy-induced neuropathy: Part 2 laboratoryassessed and objective outcomes. Pain Manag Nurs 2019;20:623-32.

34. Zhong B. How to calculate sample size in randomized controlled trial? J Thorac Dis 2009;1:51-4.

35. Bao T, Seidman AD, Piulson L, et al. A phase IIA trial of acupuncture to reduce chemotherapy-induced peripheral neuropathy severity during neoadjuvant or adjuvant weekly paclitaxel chemotherapy in breast cancer patients. Eur J Cancer 2018;101:12-9.

36. Oleson T. Auriculotherapy Manual: Chinese and Western Systems of Ear Acupuncture. London: Churchill Livingstone, 2014.

37. Jensen MP. The validity and reliability of pain measures in adults with cancer. J Pain 2003;4:2-21.

38. Paice JA, Cohen FL. Validity of a verbally administered numeric rating scale to measure cancer pain intensity. Cancer Nurs 1997;20:88-93. 
39. Cavaletti G, Frigeni B, Lanzani F, et al. The Total Neuropathy Score as an assessment tool for grading the course of chemotherapy-induced peripheral neurotoxicity: comparison with the National Cancer Institute-Common Toxicity Scale. J Peripher Nerv Syst 2007;12:210-5.

40. Cavaletti G, Cornblath DR, Merkies IS, et al. The chemotherapy-induced peripheral neuropathy outcome measures standardization study: from consensus to the first validity and reliability findings. Ann Oncol 2013;24:454-62.

41. Cavaletti G, Bogliun G, Marzorati L, et al. Grading of chemotherapy-induced peripheral neurotoxicity using the Total Neuropathy Scale. Neurology 2003;61:1297-300.

42. Postma TJ, Aaronson NK, Heimans JJ, et al. The development of an EORTC quality of life questionnaire to assess chemotherapy-induced peripheral neuropathy: the QLQ-CIPN20. Eur J Cancer 2005;41:1135-9.

43. Kim HY, Kang JH, Youn HJ, et al. Reliability and validity of the Korean Version of the European Organization for research and treatment of cancer Quality of Life Questionnaire to assess chemotherapy-induced peripheral neuropathy. J Korean Acad Nurs 2014;44:735-42.

44. Kieffer JM, Postma TJ, van de Poll-Franse L, et al. Evaluation of the psychometric properties of the EORTC chemotherapy-induced peripheral neuropathy questionnaire (QLQ-CIPN20). Qual Life Res 2017;26:2999-3010.

45. Cella DF, Tulsky DS, Gray G, et al. The Functional Assessment of Cancer Therapy scale: development and validation of the general measure. J Clin Oncol 1993;11:570-9.

46. Winstead-Fry P, Schultz A. Psychometric analysis of the Functional Assessment of Cancer Therapy-General (FACT-G) scale in a rural sample. Cancer 1997;79:2446-52.

47. Victorson D, Barocas J, Song J, et al. Reliability across studies from the functional assessment of cancer therapy-general (FACT-G) and its subscales: a reliability generalization. Qual Life Res 2008;17:1137-46.

48. Argyriou AA, Koltzenburg M, Polychronopoulos P, et al. Peripheral nerve damage associated with administration of taxanes in patients with cancer. Crit Rev Oncol Hematol 2008;66:218-28.

49. Henderson IC, Berry DA, Demetri GD, et al. Improved outcomes from adding sequential Paclitaxel but not from escalating Doxorubicin dose in an adjuvant chemotherapy regimen for patients with node-positive primary breast cancer. J Clin Oncol 2003;21:976-83.

50. Carlson K, Ocean AJ. Peripheral neuropathy with microtubule-targeting agents: occurrence and management approach. Clin Breast Cancer 2011;11:73-81.

51. Gornstein E, Schwarz TL. The paradox of paclitaxel neurotoxicity: Mechanisms and unanswered questions. Neuropharmacology 2014;76 Pt A:175-83.

52. Hershman DL, Till C, Wright JD, et al. Comorbidities and risk of chemotherapy-induced peripheral neuropathy among participants 65 years or older in Southwest Oncology Group Clinical Trials. J Clin Oncol 2016;34:3014-22.

53. Kim M, Jung MS. Effects of chemotherapy-induced peripheral neuropathy in women with breast cancer: A structural equation approach with the theory of unpleasant symptoms. Cancer Nurs 2019. [Epub ahead of print].

54. Greenlee H, Crew KD, Capodice J, et al. Randomized sham-controlled pilot trial of weekly electro-acupuncture for the prevention of taxane-induced peripheral neuropathy in women with early stage breast cancer. Breast Cancer Res Treat 2016;156:453-64.

55. Bao T, Goloubeva O, Pelser C, et al. A pilot study of acupuncture in treating bortezomib-induced peripheral neuropathy in patients with multiple myeloma. Integr Cancer Ther 2014;13:396-404.

56. Garcia MK, Cohen L, Guo Y, et al. Electroacupuncture for thalidomide/bortezomib-induced peripheral neuropathy in multiple myeloma: a feasibility study. J Hematol Oncol 2014;7:41.

57. Schroeder S, Meyer-Hamme G, Epplee S. Acupuncture for chemotherapy-induced peripheral neuropathy (CIPN): a pilot study using neurography. Acupunct Med 2012;30:4-7.

58. Yeh CH, Chien LC, Lin WC, et al. Pilot randomized controlled trial of auricular point acupressure to manage symptom clusters of pain, fatigue, and disturbed sleep in breast cancer patients. Cancer Nurs 2016;39:402-10.

59. Sparano JA, Zhao F, Martino S, et al. Long-term followup of the E1199 phase III trial evaluating the role of taxane and schedule in operable breast cancer. J Clin Oncol 2015;33:2353-60.

60. American Cancer Society (Internet). Atlanta: Treating Breast Cancer: Chemotherapy for Breast Cancer. (cited 2020 March 10). Available online: https://www.cancer.org/ cancer/breast-cancer/treatment/chemotherapy-for-breastcancer.html

61. Kim M, Chung E, Jung MS. An analytical study on research trend in auriculotherapy in Korea. J Korean Acad Fundam Nurs 2020;27:323-32.

62. Freund CL, Clayton EW, Wilfond BS. Natural settings trials--improving the introduction of clinical genetic tests. 
J Law Med Ethics 2004;32:106-10.

63. Shin J, Park H. Effects of auricular acupressure on constipation in patients with breast cancer receiving chemotherapy: A randomized control trial. West J Nurs Res 2018;40:67-83.

64. Eghbali M, Yekaninejad MS, Varaei S, et al. The effect of auricular acupressure on nausea and vomiting caused by chemotherapy among breast cancer patients. Complement Ther Clin Pract 2016;24:189-94.

65. Bhide A, Shah PS, Acharya G. A simplified guide to randomized controlled trials. Acta Obstet Gynecol Scand 2018;97:380-7.

Cite this article as: Jung MS, Lee JS, Sohn EH, Cha K, Dlamini NS, Kim M. Efficacy and safety of auricular acupressure for chemotherapy-induced peripheral neuropathy among patients with breast cancer: a study protocol for a randomized controlled trial. Ann Palliat Med 2021;10(1):721-732. doi: 10.21037/apm-20-1469 
Table S1 Checklist for items in STRICTA

\begin{tabular}{|c|c|c|}
\hline Item & Detail & \\
\hline \multirow[t]{3}{*}{$\begin{array}{l}\text { 1. Acupuncture } \\
\text { rationale }\end{array}$} & 1a) Style of acupuncture & $\begin{array}{l}\text { Auricular acupressure based on traditional Chinese medicine and the point selected by World Health } \\
\text { Organization standard }\end{array}$ \\
\hline & $\begin{array}{l}\text { 1b) Reasoning for treatment provided, based on historical context, literature } \\
\text { sources, and consensus methods, with references where appropriate }\end{array}$ & $\begin{array}{l}\text { The auricular acupressure points based on Zhang-fu theory and reflexology through literature reviews } \\
\text { with clinical recommendations of three of the Korean AA educators }\end{array}$ \\
\hline & 1c) Extent to which treatment was varied & No variation \\
\hline \multirow[t]{7}{*}{ 2. Details of needling } & $\begin{array}{l}\text { 2a) Number of needle insertions per subject per session (mean and range } \\
\text { where relevant) }\end{array}$ & 4 seeds \\
\hline & 2b) Names (or location if no standard name) of points used (uni/bilateral) & Shenmen, liver, spleen, and toe or finger points, unilateral \\
\hline & $\begin{array}{l}\text { 2c) Depth of insertion, based on a specified unit of measurement, or on a } \\
\text { particular tissue level }\end{array}$ & Not applicable \\
\hline & 2d) Response sought (e.g., de qi or muscle twitch response) & Not applicable \\
\hline & 2e) Needle stimulation (e.g., manual, electrical) & Manual acupressure \\
\hline & 2f) Needle retention time & 5 consecutive days \\
\hline & 2g) Needle type (diameter, length, and manufacturer or material) & Vaccaria seeds (black with diameters of $0.2 \mathrm{~cm})$ covered by waterproof adhesive tape $(0.7 \mathrm{~cm} \times 0.7 \mathrm{~cm})$ \\
\hline \multirow[t]{2}{*}{ 3. Treatment regimen } & 3a) Number of treatment sessions & 3 sessions \\
\hline & 3b) Frequency and duration of treatment sessions & Weekly for 3 weeks \\
\hline \multirow[t]{2}{*}{$\begin{array}{l}\text { 4. Other component of } \\
\text { treatment }\end{array}$} & $\begin{array}{l}\text { 4a) Details of other interventions administered to the acupuncture group (eg, } \\
\text { moxibustion, cupping, herbs, exercises, lifestyle advice) }\end{array}$ & No other interventions \\
\hline & $\begin{array}{l}\text { 4b) Setting and context of treatment, including instructions to practitioners, } \\
\text { and information and explanations to patients }\end{array}$ & $\begin{array}{l}\text { In a university hospital } \\
\text { This study will inform the participants about the auricular acupressure points which is not proven to } \\
\text { helpful for CIPN, but anticipated to be effective based on literature reviews. }\end{array}$ \\
\hline $\begin{array}{l}\text { 5. Practitioner } \\
\text { background }\end{array}$ & $\begin{array}{l}\text { Description of participating acupuncturists (qualification or professional } \\
\text { affiliation, years in acupuncture practice, other relevant experience) }\end{array}$ & Certified by two institutes which educate auricular acupressure in Korea \\
\hline \multirow[t]{10}{*}{$\begin{array}{l}\text { 6. control or comparator } \\
\text { interventions }\end{array}$} & $\begin{array}{l}\text { 5a) Rationale for the control or comparator in the context of the research } \\
\text { question, with sources that justify this choice }\end{array}$ & To confirm the efficacy of auricular acupressure compared to sham auricular acupressure \\
\hline & $\begin{array}{l}\text { 5b) Precise description of the control or comparator. If sham acupuncture or } \\
\text { any other type of acupuncture-like control is used, provide details as for Items } \\
1 \text { to } 3 \text { above }\end{array}$ & \\
\hline & - Style of acupuncture: & $\begin{array}{l}\text { Auricular acupressure based on traditional Chinese medicine and the point selected by World Health } \\
\text { Organization standard }\end{array}$ \\
\hline & - Names (or location if no standard name) of points used (uni/bilateral) & Tooth, tongue, jaw, and internal ear points, unilateral \\
\hline & - Number of needle insertions per subject per session & 4 seeds \\
\hline & - Needle stimulation: & Manual acupressure \\
\hline & - Needle retention time: & 5 consecutive days \\
\hline & - Needle type & Vaccaria seeds (black with diameters of $0.2 \mathrm{~cm})$ covered by waterproof adhesive tape $(0.7 \mathrm{~cm} \times 0.7 \mathrm{~cm})$ \\
\hline & - Number of treatment sessions & 3 sessions \\
\hline & - Frequency and duration of treatment sessions & weekly for 3 weeks \\
\hline
\end{tabular}

